\title{
Direct Radical Scavenging Activity of Benzbromarone Provides Beneficial Antioxidant Properties for Hyperuricemia Treatment
}

\author{
Daisuke Kadowaki, $, a, b, \#$ Shoichi Sakaguchi, ${ }^{a, \#}$ Yohei Miyamoto, ${ }^{c}$ Kazuaki Taguchi,, , \\ Nanako Muraya, ${ }^{a}$ Yuki Narita, ${ }^{a, b}$ Keizo Sato, ${ }^{e}$ Victor Tuan Giam Chuang, ${ }^{f}$ Toru Maruyama, ${ }^{b, c}$ \\ Masaki Otagiri, ${ }^{c, d}$ and Sumio Hirata ${ }^{a, b}$ \\ ${ }^{a}$ Department of Clinical Pharmacology, Faculty of Pharmaceutical Sciences, Kumamoto University; 5-1 \\ Oe-honmachi, Chuo-ku, Kumamoto 862-0973, Japan: ${ }^{b}$ Center for Clinical Pharmaceutical Sciences, Faculty \\ of Pharmaceutical Sciences, Kumamoto University; 5-1 Oe-honmachi, Chuo-ku, Kumamoto 862-0973, Japan. \\ ${ }^{c}$ Department of Biopharmaceutics, Graduate School of Pharmaceutical Sciences, Kumamoto University; 5-1 \\ Oe-honmachi, Chuo-ku, Kumamoto 862-0973, Japan: ${ }^{d}$ Faculty of Pharmaceutical Sciences, Sojo University; \\ 4-22-1 Ikeda, Nishi-ku, Kumamoto 860-0082, Japan: ${ }^{e}$ School of Pharmacy, Kyushu University of Health and \\ Welfare; 1714-1 Yoshino Nobeoka, Miyazaki 882-8508, Japan: and ${ }^{f}$ School of Pharmacy, Curtin Health Innovation \\ Research Institute, Curtin University; GPO Box U1987, Perth 6845, WA, Australia.
}

Received July 15, 2014; accepted December 22, 2014; advance publication released online January 15, 2015

Uric acid exerts an important antioxidant effect against external oxidative stress under physiological conditions. However, uric acid itself can increase oxidative stress via reduced nicotinamide adenine dinucleotide phosphate (NADPH) oxidase activation in adipocytes and vascular cells. Uric acid transporter 1 is involved in the generation of this oxidative stress. Furthermore, uric acid locally activates the renin-angiotensin system, thus producing angiotensin II and subsequently increasing intracellular oxidative stress. Benzbromarone has been reported to suppress uric acid reabsorption via uric acid transporter 1 inhibition in renal tubular cells. In this study we evaluated the in vitro antioxidant effect of benzbromarone from several perspectives. First, the direct radical-trapping capacity of benzbromarone was measured by chemiluminescence assay and electron paramagnetic resonance spectroscopy. Second, the intracellular antioxidant activity of benzbromarone in hyperuricemia was evaluated using endothelial cells. In light of these results, benzbromarone is hypothesized directly to scavenge the superoxide anion radical. In addition, benzbromarone inhibited reactive oxygen species production that was induced by angiotensin II or uric acid in endothelial cells. These findings suggest that benzbromarone possesses the ability directly to scavenge radicals and may act as an antioxidant against uric acid and angiotensin II-induced oxidative stresses in endothelial cells at therapeutically achievable levels in blood.

Key words benzbromarone; uric acid; reactive oxygen species; endothelial dysfunction

Both the kidneys and the vasculature are rich sources of reactive oxygen species (ROS), particularly superoxide $\left(\mathrm{O}_{2}^{--}\right)$, which is mainly generated by reduced nicotinamide adenine dinucleotide phosphate (NADPH) oxidase. Excessive ROS derived from NADPH oxidase are involved not only in the pathogenesis of both hypertension and renal disease $\mathrm{e}^{1-3)}$ but also in the reduction of the potent vasodilator nitric oxide $(\mathrm{NO}){ }^{4-6)}$ In this process, peroxynitrite generated by the reaction between $\mathrm{NO}$ and $\mathrm{O}_{2}^{--}$enhances further oxidation of biological substrates such as proteins, lipids and nucleic acids, ${ }^{7,8)}$ resulting in deteriorating renal dysfunction and vascular damage under pathophysiological conditions.

Uric acid plays an important role in the pathogenesis of ROS-related diseases, ${ }^{9)}$ as it acts at physiological concentrations as a powerful antioxidant that protects endothelial cells from extracellularly generated ROS. ${ }^{10)}$ However, uric acid has been reported to stimulate increases in NADPH oxidasederived ROS production in adipocytes, vascular smooth muscle cells and vascular endothelial cells. ${ }^{11,12)}$ Furthermore, a recent report suggested that uric acid also activates the local renin-angiotensin system (RAS) and that angiotensin II (AII) increases intracellular oxidative stress by stimulating NADPH oxidase. $^{13)}$ Taken together, these findings provide evidence that

\footnotetext{
\# These authors contributed equally to this work.

uric acid functions as a pro-oxidant in hyperuricemia, despite acting as an antioxidant under physiological conditions. Therefore, the presence of excessive uric acid in the bloodstream such as is seen in hyperuricemia without crystal deposition and gout, is strongly associated with ROS-related disorders, such as cardiovascular disease (CVD), chronic kidney disease (CKD), hypertension and the risk of mortality. ${ }^{14)}$

Benzbromarone is a uricosuric agent that has been used for more than 30 years in clinical practice. Benzbromarone acts by suppressing uric acid reabsorption via inhibition of the uric acid transporter 1 (URAT1) in renal tubular cells, and its maximum blood concentration $\left(C_{\max }\right)$ following $100 \mathrm{mg}$ of oral administration is approximately $2.3 \mu \mathrm{g} / \mathrm{mL}(5.4 \mu \mathrm{M})$ in healthy subjects. ${ }^{15)}$ Both benzbromarone and probenecid, a URAT1 inhibitor, have previously been reported to inhibit intracellular NADPH oxidase-derived ROS production by interfering with uric acid uptake via URAT1 in adipocytes and vascular smooth muscle cells. ${ }^{11,16)}$ Moreover, benzbromarone, in contrast to probenecid, has a phenolic hydroxy group that may be involved in its radical trapping action. Therefore, we speculate that benzbromarone may possess an additional mechanism of ROS inhibition, particularly direct ROS scavenging, along with a decrease of NADPH oxidase-derived ROS production via URAT1 inhibition. Thus, we first examined the direct radical-trapping capacity of benzbromarone using a chemilu- 
minescence assay and electron paramagnetic resonance (EPR) spectroscopy. Subsequently, the antioxidant activity of therapeutic concentrations of benzbromarone in hyperuricemia or under RAS activation was evaluated in human umbilical vein endothelial cells (HUVECs).

\section{MATERIALS AND METHODS}

Chemicals and Materials Xanthine (X), Xanthine oxidase $(\mathrm{XO})$, uric acid sodium salt, 2-amino-4-hydroxypteridine (pterin), 2-amino-4,7-dihydroxypteridine (isoxanthopterin), $t$ butyl hydroperoxide $(t-\mathrm{Bu}), \mathrm{Cu}, \mathrm{Zn}$-superoxide dismutase $(\mathrm{Cu}$, Zn-SOD), AII, Benzbromarone and allopurinol (ALP) were purchased from Sigma Chemical Co. (St. Louis, MO, U.S.A.). 2,2-Dimethyl-3,4-dihydro-2H-pyrrole-1-oxide (DMPO) was purchased from Alexis Corporation. 5-(and-6)-Chloromethyl-2', 7'-dichlorodihydrofluorescein diacetate (CM- ${ }_{2}$ DCFDA) was purchased from Invitrogen (Eugene, OR, U.S.A.). Diethylene-triamine-pentaacetic acid (DTPA) was purchased from Dojindo Laboratories (Kumamoto, Japan). Trolox was purchased from CalBiochem Inc. (San Diego, CA, U.S.A.). Luminol (99\%), $\mathrm{FeCl}_{2}$ (iron), and hemoglobin (Hb), $\mathrm{N}$-acetylcysteine (NAC) were purchased from Nacalai Tesque Inc. (Kyoto, Japan). MCDB 131, L-glutamine was purchased from GIBCO (Carlsbad, CA, U.S.A.). Ten percent fetal calf serum (FCS) was purchased from Sanko Junyaku (Tokyo, Japan). HUVECs were purchased from Dainippon Sumitomo Pharma (Osaka, Japan). All other chemicals were of the highest grade available from commercial sources.

Chemiluminescence Assay The chemiluminescence response to $\mathrm{O}_{2}^{-}$or the peroxy radical (LOO ) generated by the $\mathrm{X} / \mathrm{XO}$ or $\mathrm{Hb} / \mathrm{t}-\mathrm{Bu}$ system was continuously measured and recorded for $10 \mathrm{~min}$ at room temperature using a luminometer (MiniLumat LB 9506, Berthold Technologies, TN, U.S.A.). The reaction of the $\mathrm{X} / \mathrm{XO}$ system was initiated by adding $40 \mu \mathrm{g} / \mathrm{mL} \mathrm{X}$ to phosphate buffered saline (PBS; $\mathrm{pH} 7.4$ ) containing $0.004 \mathrm{U} / \mathrm{mL} \mathrm{XO}, 250 \mu \mathrm{M}$ DTPA and $500 \mu \mathrm{m}$ luminol in the absence or presence of benzbromarone. $\mathrm{Cu}, \mathrm{Zn}-\mathrm{SOD}(5 \mathrm{U} /$ $\mathrm{mL}$ ) was added to the above mixture to confirm the specificity of the reaction. The reaction of the $\mathrm{Hb} / t$ - $\mathrm{Bu}$ system was initiated by adding $0.1 \mathrm{~mm} t$-Bu to the PBS containing $4 \mu \mathrm{g} / \mathrm{mL}$ $\mathrm{Hb}$, and $500 \mu \mathrm{M}$ luminal in the absence or presence of benzbromarone. To confirm the specificity of the reaction, $100 \mu \mathrm{M}$ Trolox was used as a positive control and was added to the above mixture. The activity of XO was determined by monitoring the conversion of $\mathrm{X}$ to uric acid, according to a previous report. ${ }^{17)}$ ALP was used to confirm the positive reaction.

EPR Spectroscopy Superoxide concentrations from human neutrophils were determined using EPR spin trapping with EMPO, and the scavenging activity was calculated from the relative peak intensities corresponding to the EMPO-superoxide adduct (EMPO-OOH) EPR signal. Human neutrophils $\left(1.0 \times 10^{6}\right.$ cells $\left./ \mathrm{mL}\right)$ were pre-treated with phorbol myristate acetate $(10 \mathrm{ng} / \mathrm{mL})$ for $7 \mathrm{~min}$ at $37^{\circ} \mathrm{C}$ to activate the cells. Aliquots of this cell suspension were combined with $100 \mu \mathrm{M}$ DTPA and $25 \mathrm{~mm}$ EMPO in Hank's Balanced Salt Solution (HBSS) in the absence or presence of varying benzbromarone concentrations and were immediately transferred to quartz EPR flat cells. EPR spectra were recorded at room temperature on an EPR spectrometer (JES-TE 200, JEOL Ltd., Tokyo, Japan) under the following conditions: modula- tion frequency, $100 \mathrm{kHz}$; modulation amplitude, 50 Gauss; scanning field, $335.2 \pm 5 \mathrm{mT}$; receiver gain, 300-500; response time, $0.03 \mathrm{~s}$; sweep time, $2 \mathrm{~min}$; microwave power, $40 \mathrm{~mW}$; and microwave frequency, $9.43 \mathrm{GHz}$. $\mathrm{Cu}, \mathrm{Zn}-\mathrm{SOD}(5 \mathrm{U} / \mathrm{mL})$ was used as a positive control and was added to the above mixture to confirm the reaction. After recording the EPR spectra, the signal intensities of the EMPO-OOH adducts were normalized against that of a manganese oxide $\left(\mathrm{Mn}^{2+}\right)$ signal, in which $\mathrm{Mn}^{2+}$ served as an internal control.

Cell Culture HUVECs were cultured in MCDB131 medium containing 5\% FCS under standard cell culture conditions (humidified atmosphere, $5 \% \mathrm{CO}_{2}, 37^{\circ} \mathrm{C}$ ). HUVECs were seeded to collagen-coated tissue culture flasks and further grown to confluence until used in cellular assays. HUVECs were used in experiments at passages between 3 and 5 .

Measurement of ROS Production To measure the production of ROS, CM- $\mathrm{H}_{2}$ DCFDA, a ROS-sensitive fluorescent dye, was used as a ROS probe. HUVECs were cultured in 96well plates $\left(8 \times 10^{3}\right.$ cells/well $)$ at $37^{\circ} \mathrm{C}$ for $24 \mathrm{~h}$, and then incubated with HBSS containing $5 \mu \mathrm{M} \mathrm{CM}-\mathrm{H}_{2}$ DCFDA for $30 \mathrm{~min}$ to incorporate $\mathrm{CM}-\mathrm{H}_{2} \mathrm{DCFDA}$ into the cell. After removal of HBSS and rinsing CM- $\mathrm{H}_{2}$ DCFDA from the wells, the cells were incubated with benzbromarone $(1.2-23.4 \mu \mathrm{M})$ in medium for $30 \mathrm{~min}$ and then added to $50 \mu \mathrm{M}$ AII or $536 \mu \mathrm{M}(0.9 \mathrm{mg} / \mathrm{dL})$ uric acid. Mean fluorescence intensity (MFI) was measured as intracellular ROS production (excitation $485 \mathrm{~nm}$ and emission $535 \mathrm{~nm}$ ) after $1 \mathrm{~h}$ using a fluorescence microplate reader (SPECTRA FLUOR, TECAN Ltd., Männedorf, Switzerland). NAC was used as a positive control.

Viability of HUVECs HUVECs were incubated in 96well plates $\left(8 \times 10^{4}\right.$ cells $\left./ \mathrm{mL}\right)$ at $37^{\circ} \mathrm{C}$ for $24 \mathrm{~h}$. The culture medium was then removed and washed twice with HBSS. HUVECs were added to benzbromarone, and then incubated with uric acid for $24 \mathrm{~h}$. Cell viability was determined after $24 \mathrm{~h}$ post treatment growth in 96-well culture plates with the Cell Counting Kit-8 (Dojindo Laboratories, Kumamoto, Japan) assays according to the manufacturer's instructions. NAC was used as a positive control.

Statistical Analysis The results are reported as the mean \pm S.D. Among $2>$ mean values, statistical significance was evaluated by Tukey-Kramer. Bonferroni's test was used for EPR data. For all analyses, $p<0.05$ was regarded as being statistically significant.

\section{RESULTS}

Benzbromarone Scavenges $\mathrm{O}_{2}^{--}$and $\mathrm{LOO}^{-}$Using Chemiluminescence Assay The antioxidant activity of benzbromarone against $\mathrm{O}_{2}^{--}$generated by the $\mathrm{X} / \mathrm{XO}$ system was examined using chemiluminescence assay. As shown in Fig. 1A, benzbromarone decreased the chemiluminescence responses to $\mathrm{O}_{2}^{--}$at concentrations of $1.2-23.4 \mu \mathrm{M}$. In addition, the amount of uric acid, the metabolite of $\mathrm{X}$ in this system, was not affected by benzbromarone, indicating that benzbromarone, unlike the $\mathrm{XO}$ inhibitor allopurinol, has no effect on $\mathrm{XO}$ activity (Fig. 1B). Similar experiments were also performed using the $\mathrm{Hb} / t$-Bu system, which mainly generated LOO . As a result, chemiluminescence response with $\mathrm{LOO}$ was also decreased by benzbromarone (Fig. 1C).

Benzbromarone Scavenges $\mathrm{O}_{2}^{--}$Derived from Polymorphonuclear Neutrophils Using EPR Spectroscopy The 

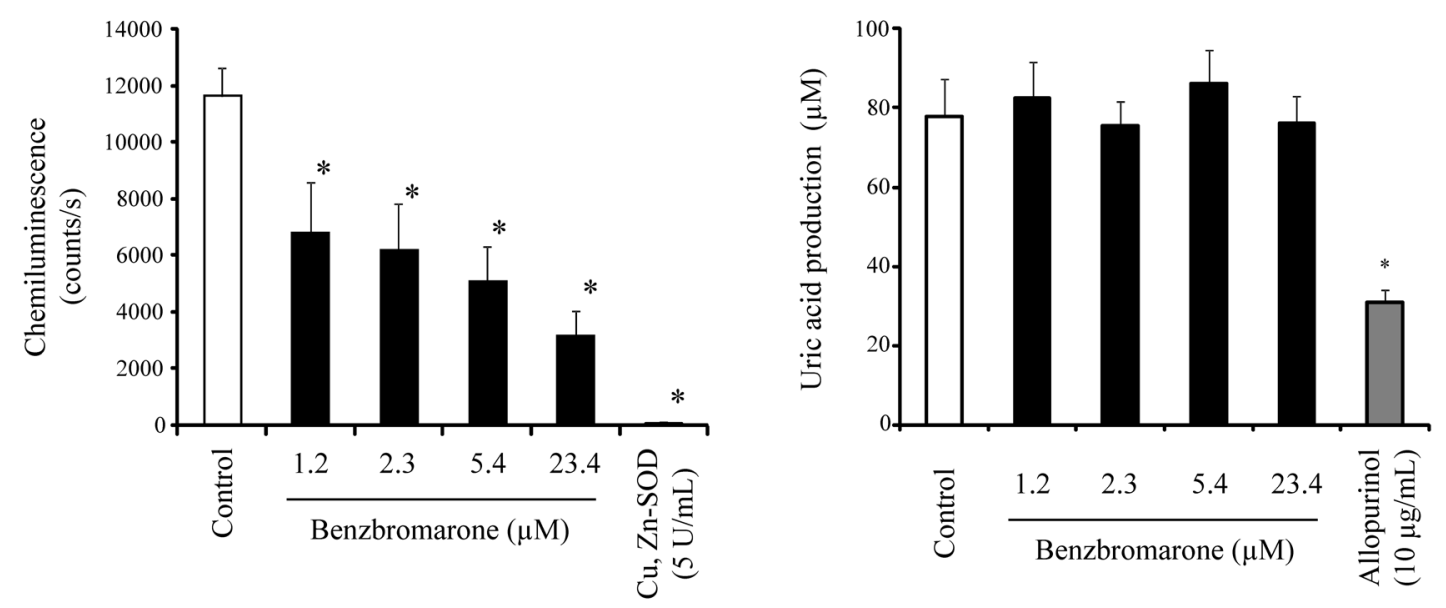

C)

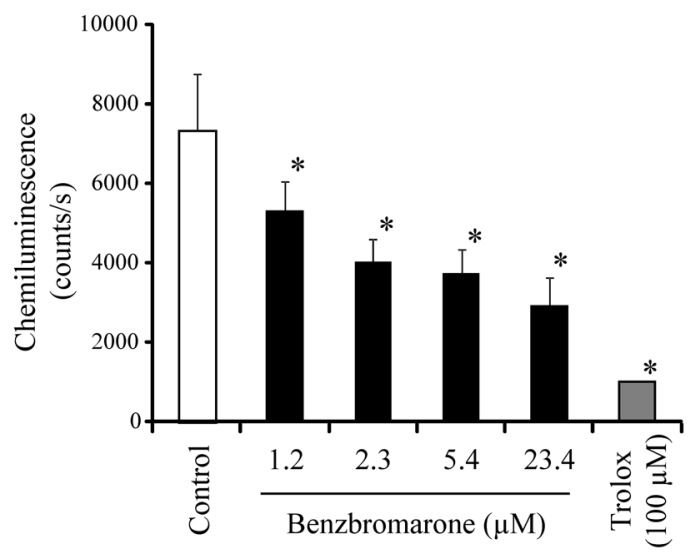

Fig. 1. Benzbromarone Scavenges $\mathrm{O}_{2}^{--}$and LOO by the $\mathrm{X} / \mathrm{XO}$ or $\mathrm{Hb} / \mathrm{t}-\mathrm{Bu}$ Systems

A) Benzbromarone decreases the luminol-dependent chemiluminescence response induced by the X/XO system. B) Effect of benzbromarone on uric acid production. C) Benzbromarone decreases the luminol-dependent chemiluminescence response induced by the $\mathrm{Hb} / t$-Bu system. Values are expressed as the mean $\pm \mathrm{S}$. D. $(n=4-7)$. ${ }^{*} p<0.01$ in comparison to the control.

scavenging activity of benzbromarone against $\mathrm{O}_{2}^{--}$was also examined in a cell system. Benzbromarone scavenged $\mathrm{O}_{2}^{-}$ derived from phorbol myristate acetate-stimulated human neutrophils (Figs. 2A, B). Moreover, benzbromarone exerted radical scavenging activity at a lower concentration than $C_{\max }$ $(5.4 \mu \mathrm{M})$.

Effect of Benzbromarone on Intracellular Uric AcidInduced ROS Production and Cell Viability in HUVECs Next, the intracellular antioxidant activity of benzbromarone in hyperuricemia was evaluated using human endothelial cells. Uric acid induced oxidative stress at $536 \mu \mathrm{M}$, and benzbromarone significantly inhibited uric acid-induced intracellular ROS production under pathophysiological conditions in HUVECs (Fig. 3A). In association with ROS production, the decreased viability of HUVECs by uric acid treatment $(536 \mu \mathrm{M})$ was improved by incubation with benzbromarone (Fig. 3B).

Effect of Benzbromarone on AII-Induced Intracellular ROS Production in HUVECs As described in the introduc- tion, uric acid activates the local RAS, leading to the production of AII. ${ }^{13)}$ Therefore, we examined the effect of benzbromarone on the intracellular ROS production stimulated by AII in HUVECs. When AII was added to HUVECs, a significant increase in the fluorescence intensity was observed. However, the fluorescence intensity was decreased by the co-treatment of benzbromarone at concentrations approximately equal to $C_{\max }$ (Fig. 4).

\section{DISCUSSION}

When uric acid is taken up by adipocytes, $\mathrm{O}_{2}^{-}$synthesis is catalyzed by NADPH oxidase and lipid radicals. ${ }^{11)}$ Furthermore, excessive AII production, as a result of RAS activation by uric acid, leads to collapse of the intracellular redox state and cause the induction of apoptosis and growth inhibition of the endothelial cells. ${ }^{13)}$ In the present study, we first revealed that benzbromarone, an antihyperuricemic drug, possesses direct scavenging ability against $\mathrm{O}_{2}^{-}$and $\mathrm{LOO}^{-}$within clini- 
A)

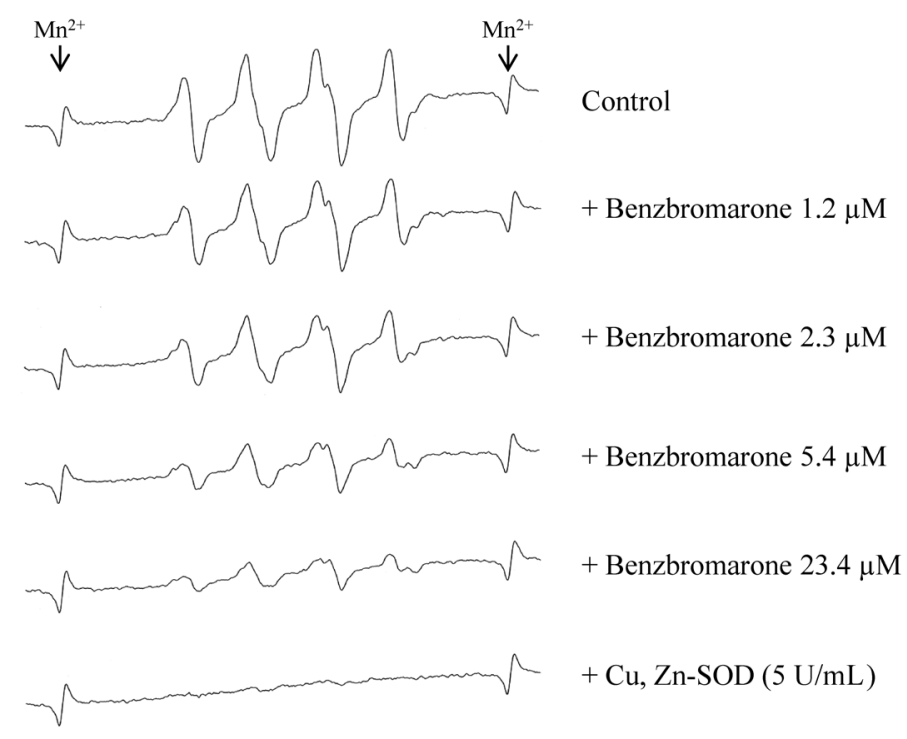

B)

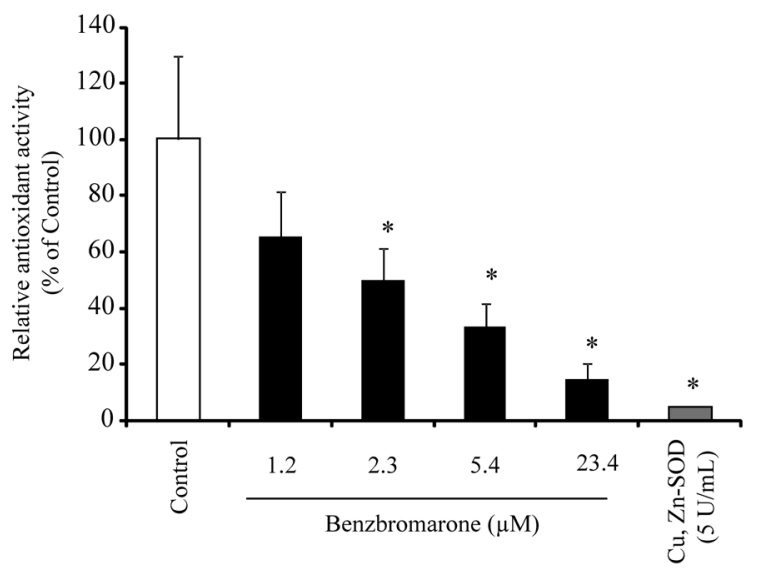

Fig. 2. Antioxidant Properties of Benzbromarone to Phorbol Myristate Acetate-Stimulated Neutrophils as Assessed by EPR Spectroscopy A) EPR spectrum of DMPO spin adducts of $\mathrm{O}_{2}^{--}$generated in phorbol myristate acetate-stimulated neutrophils; B) Quantitation of the $\mathrm{O}_{2}^{-}$concentration. Values are expressed as the mean \pm S.D. $(n=3)$. ${ }^{*} p<0.01$ in comparison to the control.

A)

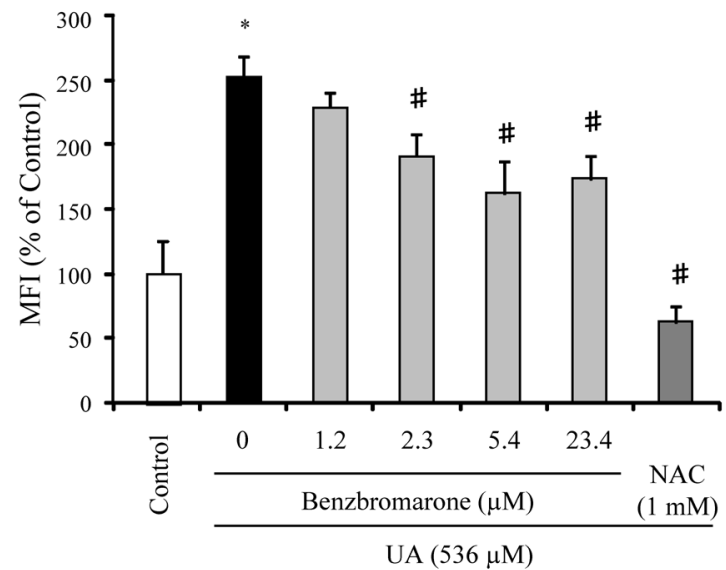

B)

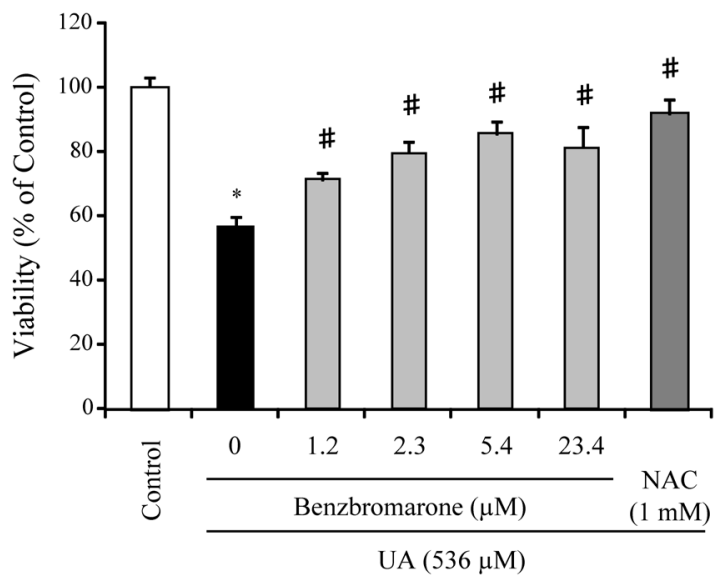

Fig. 3. Effects of Benzbromarone or Probenecid on Uric Acid-Derived ROS Production and Cell Viability in HUVECs

Effects of benzbromarone on intracellular ROS production A) or on the cytotoxicity B) by uric acid. Values are expressed as the mean \pm S.D. $(n=4)$. ${ }^{*}<<0.01$ in comparison to the control. ${ }^{\#} p<0.01$ in comparison to uric acid only. 


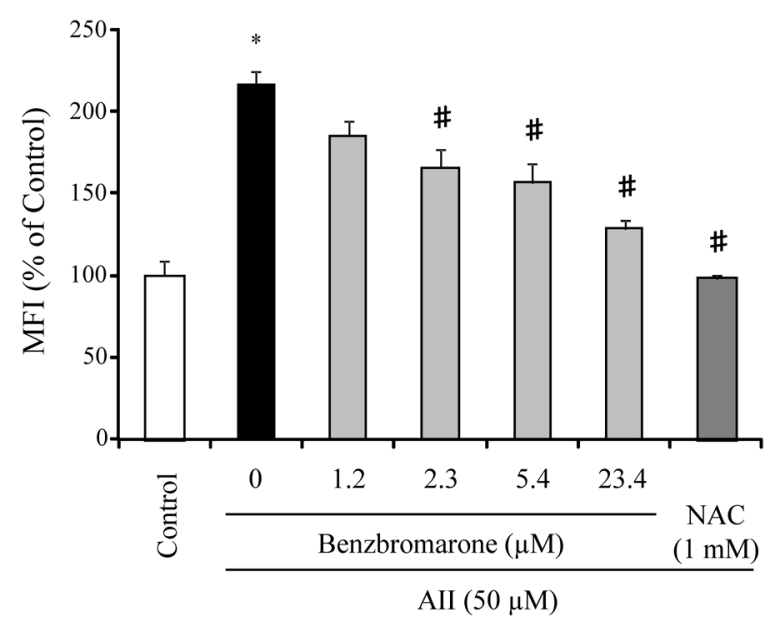

Fig. 4. Effects of Benzbromarone on Intracellular ROS Production Induced by AII in HUVECs

Values are expressed as the mean \pm S.D. $(n=4)$. ${ }^{*} p<0.01$ in comparison to control. ${ }^{\#} p<0.01$ in comparison to AII only.

cally therapeutic concentrations. In addition, benzbromarone suppressed ROS production induced by uric acid and AII in vascular endothelial cells. The direct and indirect antioxidant activities of benzbromarone are thought to exert a protective effect on cells and tissues against ROS-derived damage.

It is well known that $\mathrm{O}_{2}^{--}$is key in initiating the production of various ROS, such as hydroxyl radicals and peroxynitrite, under physiological conditions. In addition, $\mathrm{O}_{2}^{--}$mediates pro-oxidative and proinflammatory changes in endothelial cells. This $\mathrm{O}_{2}^{--}$-induced damage can disrupt the balance between vasodilation and vasoconstriction, which affects arterial remodeling and inflammatory mediators. Therefore, oxidative stress derived from $\mathrm{O}_{2}^{--}$may cause cumulative injuries to the endothelium and consequently, may be involved in the pathogenesis of cardiovascular disease, hypertension and kidney disease. ${ }^{18,19)}$ Our study clearly showed that benzbromarone is a potent scavenger of $\mathrm{O}_{2}^{--}$generated from both the $\mathrm{X} / \mathrm{XO}$ system (Fig. 1A) and activated neutrophils (Fig. 2). Furthermore, benzbromarone prevented cell death related to intracellular ROS production (Fig. 3). Hyperuricemia is strongly associated with CVD and CKD and the increased risk of mortality. ${ }^{14)}$ Benzbromarone may exert a stabilizing effect on CKD and CVD. In fact, a retrospective study reported that patients with renal impairment (CKD stage 3 or higher) receiving benzbromarone monotherapy did not observe a further deterioration in renal function. $^{20)}$

As described above, benzbromarone directly scavenges ROS, such as $\mathrm{O}_{2}^{--}$and LOO, at clinical concentrations (Figs. 1,2). Therefore, the mechanism of ROS suppression could be explained in part by the direct interaction of benzbromarone with $\mathrm{O}_{2}^{-}$. Recently, Anzai and Endou reported that $\mathrm{IC}_{50}$ for benzbromarone against URAT1 was $0.05 \mu \mathrm{M}^{21)}$ In addition, it was reported that benzbromarone partially inhibited voltagedriven urate transporter 1 (URATv1), the extracellular uric acid efflux transporter, in the renal tubular cell. ${ }^{22)}$ Considering these reports and our data that $1.2 \mu \mathrm{M}$ of benzbromarone inhibited ROS by approximately $10 \%$, benzbromarone might affect both URAT1 and URATv1. Further studies are necessary to clarify the antioxidant pathway of benzbromarone in greater detail.

Recently, uric acid has been reported to induce a substan- tial oxidative burst at physiological concentrations $(357 \mu \mathrm{M}$ or more), which was associated with local RAS activation, AII production and the induction of senescence and apoptosis of human endothelial cells. ${ }^{13)}$ Indeed, both hyperuricemic and AII-infused rats developed hypertension, afferent arteriolopathy, glomerular hypertrophy and interstitial inflammation in the kidney. ${ }^{23-25)}$ In this study using HUVECs, for the first time, we have demonstrated the protective effects of benzbromarone against AII-derived ROS (Fig. 4). Furthermore, a good correlation was observed between cell viability and oxidative stress ( $r=0.937, p<0.01$, data not shown). Therefore, the antioxidant activity of benzbromarone is thought to be responsible for its cytoprotective effects. Benzbromarone reduces ROS generated by local activation of the RAS in hyperuricemia and may be a useful uricosuric agent against cardiovascular disease and hypertension.

In Japan and a number of other countries, benzbromarone and allopurinol are widely used for the treatment of hyperuricemia. Allopurinol inhibits ROS production by working as an $\mathrm{XO}$ inhibitor but not as a radical scavenger, ${ }^{26)}$ whereas benzbromarone decreases ROS production directly and indirectly by working as both a URAT1 inhibitor and radical scavenger, respectively. Moreover, although oxypurinol, the active metabolite of allopurinol, causes severe side effects such as toxic epidermal necrosis or agranulocytosis in hyperuricemic patients with renal failure, ${ }^{27,28)}$ benzbromarone was reported to increase the renal clearance of oxypurinol via URAT1 inhibition in the renal tubular reabsorption of oxypurinol. ${ }^{29)}$ Therefore, the combination of benzbromarone with allopurinol is useful for achieving better therapeutic effects in treating hyperuricemia and associated diseases such as CVD or CKD due to its effective oxidative stress-reducing ability and by alleviating the side effects of oxypurinol.

Acknowledgments This work was supported in part by Grants from JSPS (KAKENHI 24790160). The Kidney Foundation, Japan (JFK 10-4), and the KUMAYAKU Alumni Research Fund.

Conflict of Interest The authors declare no conflict of interest.

\section{REFERENCES}

1) Shah SV. Oxidants and iron in chronic kidney disease. Kidney Int. Suppl., 66 (s91), S50-S55 (2004).

2) Wilcox CS. Oxidative stress and nitric oxide deficiency in the kidney: a critical link to hypertension? Am. J. Physiol. Regul. Integr. Comp. Physiol., 289, R913-R935 (2005).

3) Wilcox CS, Gutterman D. Focus on oxidative stress in the cardiovascular and renal systems. Am. J. Physiol. Heart Circ. Physiol., 288, H3-H6 (2005).

4) Gill PS, Wilcox CS. NADPH oxidases in the kidney. Antioxid. Redox Signal., 8, 1597-1607 (2006).

5) Touyz RM. Reactive oxygen species, vascular oxidative stress, and redox signaling in hypertension: what is the clinical significance? Hypertension, 44, 248-252 (2004).

6) Wilcox CS. Reactive oxygen species: roles in blood pressure and kidney function. Curr. Hypertens. Rep., 4, 160-166 (2002).

7) Takahashi MA, Asada K. Superoxide anion permeability of phospholipid membranes and chloroplast thylakoids. Arch. Biochem. Biophys., 226, 558-566 (1983). 
8) Lynch RE, Fridovich I. Effects of superoxide on the erythrocyte membrane. J. Biol. Chem., 253, 1838-1845 (1978).

9) Ames BN, Cathcart R, Schwiers E, Hochstein P. Uric acid provides an antioxidant defense in humans against oxidant- and radical-caused aging and cancer: a hypothesis. Proc. Natl. Acad. Sci. U.S.A., 78, 6858-6862 (1981).

10) Kuzkaya N, Weissmann N, Harrison DG, Dikalov S. Interactions of peroxynitrite with uric acid in the presence of ascorbate and thiols: implications for uncoupling endothelial nitric oxide synthase. Biochem. Pharmacol., 70, 343-354 (2005).

11) Sautin YY, Nakagawa T, Zharikov S, Johnson RJ. Adverse effects of the classic antioxidant uric acid in adipocytes: NADPH oxidasemediated oxidative/nitrosative stress. Am. J. Physiol. Cell Physiol., 293, C584-C596 (2007).

12) Corry DB, Eslami P, Yamamoto K, Nyby MD, Makino H, Tuck ML. Uric acid stimulates vascular smooth muscle cell proliferation and oxidative stress via the vascular renin-angiotensin system. $J$. Hypertens., 26, 269-275 (2008).

13) Yu MA, Sanchez-Lozada LG, Johnson RJ, Kang DH. Oxidative stress with an activation of the renin-angiotensin system in human vascular endothelial cells as a novel mechanism of uric acid-induced endothelial dysfunction. J. Hypertens., 28, 1234-1242 (2010).

14) Johnson RJ, Kang DH, Feig D, Kivlighn S, Kanellis J, Watanabe S, Tuttle KR, Rodriguez-Iturbe B, Herrera-Acosta J, Mazzali M. Is there a pathogenetic role for uric acid in hypertension and cardiovascular and renal disease? Hypertension, 41, 1183-1190 (2003).

15) Enomoto A, Kimura H, Chairoungdua A, Shigeta Y, Jutabha P, Cha SH, Hosoyamada M, Takeda M, Sekine T, Igarashi T, Matsuo H, Kikuchi Y, Oda T, Ichida K, Hosoya T, Shimokata K, Niwa T, Kanai Y, Endou H. Molecular identification of a renal urate anion exchanger that regulates blood urate levels. Nature, 417, 447-452 (2002).

16) Kang DH, Han L, Ouyang X, Kahn AM, Kanellis J, Li P, Feng L, Nakagawa T, Watanabe S, Hosoyamada M, Endou H, Lipkowitz M, Abramson R, Mu W, Johnson RJ. Uric acid causes vascular smooth muscle cell proliferation by entering cells via a functional urate transporter. Am. J. Nephrol., 25, 425-433 (2005).

17) Miyamoto Y, Iwao Y, Tasaki Y, Sato K, Ishima Y, Watanabe H, Kadowaki D, Maruyama T, Otagiri M. The uremic solute indoxyl sulfate acts as an antioxidant against superoxide anion radicals under normal-physiological conditions. FEBS Lett., 584, 2816-2820 (2010).

18) Harrison D, Griendling KK, Landmesser U, Hornig B, Drexler H. Role of oxidative stress in atherosclerosis. Am. J. Cardiol., 91 (3A), 7A-11A (2003).
19) Lassègue B, Griendling KK. Reactive oxygen species in hypertension; An update. Am. J. Hypertens., 17, 852-860 (2004).

20) Fujimori S, Ooyama $\mathrm{K}$, Ooyama $\mathrm{H}$, Moromizato $\mathrm{H}$. Efficacy of benzbromarone in hyperuricemic patients associated with chronic kidney disease. Nucleosides Nucleotides Nucleic Acids, 30, 10351038 (2011).

21) Anzai N, Endou H. Urate transporters: an evolving field. Semin. Nephrol., 31, 400-409 (2011).

22) Anzai N, Ichida K, Jutabha P, Kimura T, Babu E, Jin CJ, Srivastava S, Kitamura K, Hisatome I, Endou H, Sakurai H. Plasma urate level is directly regulated by a voltage-driven urate efflux transporter URATv1 (SLC2A9) in humans. J. Biol. Chem., 283, 26834-26838 (2008).

23) Mazzali M, Hughes J, Kim YG, Jefferson JA, Kang DH, Gordon KL, Lan HY, Kivlighn S, Johnson RJ. Elevated uric acid increases blood pressure in the rat by a novel crystal-independent mechanism. Hypertension, 38, 1101-1106 (2001).

24) Mazzali M, Kanellis J, Han L, Feng L, Xia YY, Chen Q, Kang DH, Gordon KL, Watanabe S, Nakagawa T, Lan HY, Johnson RJ. Hyperuricemia induces a primary renal arteriolopathy in rats by a blood pressure-independent mechanism. Am. J. Physiol. Renal Physiol., 282, F991-F997 (2002).

25) Nakagawa T, Mazzali M, Kang DH, Kanellis J, Watanabe S, Sanchez-Lozada LG, Rodriguez-Iturbe B, Herrera-Acosta J, Johnson RJ. Hyperuricemia causes glomerular hypertrophy in the rat. $\mathrm{Am} . J$. Nephrol., 23, 2-7 (2003).

26) Ohno I, Okabe H, Yamaguchi Y, Saikawa H, Uetake D, Hikita M, Gomi H, Ichida K, Hosoya T. Usefulness of combination treatment using allopurinol and benzbromarone for gout and hyperuricemia accompanying renal dysfunction: kinetic analysis of oxypurinol. Nippon Jinzo Gakkai Shi, 50, 506-512 (2008).

27) Doehner W, Schoene N, Rauchhaus M, Leyva-Leon F, Pavitt DV, Reaveley DA, Schuler G, Coats AJ, Anker SD, Hambrecht R. Effects of xanthine oxidase inhibition with allopurinol on endothelial function and peripheral blood flow in hyperuricemic patients with chronic heart failure: results from 2 placebo-controlled studies. Circulation, 105, 2619-2624 (2002).

28) Farquharson CA, Butler R, Hill A, Belch JJ, Struthers AD. Allopurinol improves endothelial dysfunction in chronic heart failure. Circulation, 106, 221-226 (2002).

29) Iwanaga T, Kobayashi D, Hirayama M, Maeda T, Tamai I. Involvement of uric acid transporter in increased renal clearance of the xanthine oxidase inhibitor oxypurinol induced by a uricosuric agent, benzbromarone. Drug Metab. Dispos., 33, 1791-1795 (2005). 